OPEN ACCESS

Edited by:

Åke Sjöholm,

Gävle Hospital, Sweden

Reviewed by:

Johnny Ludvigsson,

Linköping University, Sweden

Majid Jaberi-Douraki,

Kansas State University, United States

*Correspondence:

Zhiguang Zhou

zhouzhiguang@csu.edu.cn

Xia $\mathrm{Li}$

lixia@csu.edu.cn

Specialty section: This article was submitted to

Clinical Diabetes,

a section of the journal

Frontiers in Endocrinology

Received: 18 July 2019 Accepted: 11 November 2019

Published: 03 December 2019

Citation:

Tang R, Zhong T, Wu C, Zhou Z and Li X (2019) The Remission Phase in

Type 1 Diabetes: Role of

Hyperglycemia Rectification in Immune Modulation.

Front. Endocrinol. 10:824. doi: 10.3389/fendo.2019.00824

\section{The Remission Phase in Type 1 Diabetes: Role of Hyperglycemia Rectification in Immune Modulation}

\author{
Rong Tang ${ }^{1,2}$, Ting Zhong ${ }^{1,2}$, Chao $\mathrm{Wu}^{1,2}$, Zhiguang Zhou ${ }^{1,2 *}$ and Xia $\mathrm{Li}^{1,2 *}$ \\ ${ }^{1}$ Department of Metabolism and Endocrinology, The Second Xiangya Hospital, Central South University, Changsha, China, \\ ${ }^{2}$ Key Laboratory of Diabetes Immunology, Ministry of Education, National Clinical Research Center for Metabolic Disease, \\ Central South University, Changsha, China
}

The remission phase (or honeymoon period) is a spontaneous "temporary cure stage" in type 1 diabetes course, which provides a good human model for studying $\beta$-cell protection. The exact mechanisms are still uncertain, but one of the generally recognized mechanisms is that correction of "glucotoxicity" by exogenous insulin therapy leads to " $\beta$-cell rest" and $\beta$-cell recovery. Beyond this, the remission phase is accompanied by changes in various immune cells and immune molecules, indicating downregulation of immune response, and induction of immune tolerance. The role of hyperglycemia rectification in the regulation of immune response should be emphasized because glucose metabolism is critical to maintain the normal function of immune system. Here, recent evidence of immune modulation based on the rectification of hyperglycemia from multiple aspects such as immune cells, inflammatory cytokines, biomolecules, and cell antigenicity was reviewed. It should be noteworthy that the interaction between glucose metabolism and immune plays an important role in the pathogenesis of the remission phase. The best intervention strategy may be the combination of strict glycemic control and immune modulation to protect $\beta$-cell function as early as possible.

Keywords: type 1 diabetes, remission phase, the honeymoon period, immune modulation, hyperglycemia

\section{INTRODUCTION}

Type 1 diabetes mellitus (T1DM) is an autoimmune T-cell-mediated damage to pancreatic $\beta$ cells, leading to an absolute deficiency in endogenous insulin. Because of the irreversibility of $\beta$-cell function decay, continuous exogenous insulin treatment is essential (1). To restore the patient's $\beta$-cell function and become insulin independent is the highest goal that scholars have been pursuing for decades. Actually, the primary endpoint of T1DM intervention study is $\beta$-cell function protection. Many attempts and strategies have been tried; some have made major progress in immunotherapy, but curing T1DM is still a great challenge to be overcome (2).

During the disease process, some patients have a very special period with tempory recovery of $\beta$-cell function, which is called remission phase or "honeymoon period" (3). The concept of "remission phase" was first described by Jackson et al. (4). They observed a rapid decline in demand for exogenous insulin in diabetic children after regular insulin treatment, and hypoglycemic shock was a frequent occurrence during this period. In 1944, Brush (5) gave a more specific description of this phenomenon. Animal experiments also proved that hyperglycemia is a major factor to promote the development of diabetes, and early correction of hyperglycemia is 
conducive to the recovery of islet function $(6,7)$. Several subsequent studies confirmed that insulin treatment leads to preservation of residual $\beta$-cell function $(8,9)$.

The remission phase is a short "clinical cure stage," which provides a decent human model for studying $\beta$-cell protection and immune modulation. In-depth studies of the mechanisms of this period may lay a foundation for inhibiting autoimmune response and even achieving clinical cures for T1DM. The fundamental pathophysiological change of the remission phase is a transient recovery of $\beta$-cell function (10). The exact mechanisms are still uncertain, but one of the generally recognized mechanisms is that correction of "glucotoxicity" by exogenous insulin therapy leads to $\beta$-cell rest. Recent evidence suggested that hyperglycemia rectification might protect $\beta$-cell function through other pathways, such as inducing immune modulation $(11,12)$. The latest study found that the remission phase was accompanied by changes in the frequency of regulatory $\mathrm{T}$ cells ( $\mathrm{T}$ reg), CD $25^{+} \mathrm{CD} 127^{\text {hi }}$ cells, memory T cells, Th1 cells, B-cell subsets, neutrophils, and other immune cells (13). Besides the immune cells, a variety of cellular inflammatory factors, immune related molecules may also be related to the remission phase. This review will discuss the possible mechanisms of immune modulation of the remission phase from the aspect of cellular, inflammatory, and molecular levels and focus on the glyco-metabolism in immune cells.

\section{IMMUNE CELLS}

\section{T Cell Subsets}

The frequency of some T-cell subsets is closely related to the remission phase, and the correlation might be partly attributable to good glycemic control. Moya et al. (14) found that the high frequency of activated Treg cells (aTreg), CD45RO ${ }^{+}$memory T cells, and $\mathrm{CD} 25^{+} \mathrm{CD} 127^{\text {hi }}$ cells in T1DM patients had a positive correlation with the duration of remission phase, and these correlations only existed in the condition of satisfactory glycemic control. A study in 2018 also found that the remission phase was accompanied by dynamic changes in Treg cells, Th1 and Tc1cell subsets (13), with the decreased number of Treg cells and increased Th1 and Tc1 cells at the end of the remission phase. A recent study that examined a variety of immune cell subsets and cellular molecules in newly diagnosed children with T1DM found that the level of memory-regulatory $\mathrm{T}$ cells (mTreg) decreased after the appearance of clinical symptoms and aTreg and Th17 cells were significantly elevated in the first year of onset (the remission phase often occur) (15). The mTregs were also noted to be positively correlated with C-peptide in T1DM patients (16). Evidence of the obvious dynamic changes in Tregs in the different stages of remission phase suggested that the immune response during the remission phase was most likely related to the frequency and function of Tregs.

Treg cells $\left(\mathrm{CD} 4^{+} \mathrm{CD} 25^{+} \mathrm{T}\right.$ cells) play a critical role in the induction and maintenance of peripheral tolerance (17-19). Dysfunction of Treg cells has been found to be one of the key pathogenesis of autoimmune diabetes $(20,21)$. There is one study that showed that presence of Tregs to Teffs dominance is the underlying cause of the remission phase (22). Considering the crucial roles of glucose metabolism in T-cell function, it has been expected that hyperglycemia rectification may be a trigger event for dominance of Tregs in the remission phase.

As early as in 2008, Maciver et al. (23) found that the energy that is supplied by glucose is necessary for $\mathrm{T}$ cells to sustain growth, proliferation, and immune activity. T cells cannot produce the appropriate cytokines, such as IFN- $\gamma$, to exert an immune effect without adequate glucose uptake (23). This suggests that the immune function of $\mathrm{T}$ cells requires a normal state of glucose metabolism. Treg cells have also been implicated in glucose metabolism in numerous studies $(24,25)$. Recent study showed that the growth and differentiation processes of Tregs and Th17 are significantly dependent on glycolysis and aerobic phosphorylation (26). In this regard, although there is no direct evidence available, it is rational to deduce that rapid rectification of hyperglycemia at the initial stage of T1DM might help to recover the impaired function of Treg cells and downregulate the immune response that is characterized by remission phase. Indeed, in the study of Moya $\mathrm{R}$, the frequent changes of aTreg, CD $25^{+} \mathrm{CD} 127^{\text {hi }}$ cells, and CD $45 \mathrm{RO}^{+}$memory cells were correlated with better blood glucose control (14), which may serve as indirect evidence for this hypothesis.

In addition to Tregs, other T-cell subsets are less reported in the remission phase. In the previously mentioned study, Moya showed that the $\mathrm{CD} 25^{+} \mathrm{CD} 127^{\text {hi }}$-cell frequency was significantly correlated with that of aTregs. In this way, it could be speculated that good glycemic control might also restore the function of $\mathrm{CD} 25^{+} \mathrm{CD} 127^{\text {hi }}$ cells, which promote the development of Tr1 cells through CD44 and CD44v6 signaling pathways (14). $\mathrm{Tr} 1$ and memory $\mathrm{T}$ cells have also been demonstrated to inhibit autoreactive inflammation $(27,28)$, but their roles in the remission phase have not been reported so far.

\section{B-Cell Subsets}

Recent studies have shown that B lymphocytes also play an indispensable role in the pathogenesis of the autoimmune diabetes $(29,30)$. A study showed that the frequency changes of marginal zone B cells (MZB), follicular B cells (FoB), and other Bcell subsets were associated with T1DM, and the regulatory B cells (Breg) have immunomodulatory effects (30). Limited reports showed that the remission phase was accompanied by changes in B-cell frequency $(13,15)$. Glucose metabolism had also been shown to play an important role in the normal functioning of B cells. Therefore, it may be hypothesized that hyperglycemia rectification during the remission phase affects the frequency of B cells.

Studies on the relationship between B-cell subsets and the remission phase are rare. In 2018, Fitas et al. (13) found that the absolute number and relative frequency of $\mathrm{B}$ cells decreased significantly during the disease course when compared with the onset stage and hit the lowest level in the remission phase. A recent study found that the Breg cells significantly increased during the remission phase (15). In addition, the study showed that the intervention of the anti-CD20 monoclonal antibody rituximab can extend the duration of the remission phase to 2 years (31). These combined results suggested that B lymphocytes are involved in the pathogenesis of the remission phase. 
Among all B-cell subsets, the Breg subpopulation with immunomodulatory effects is a research hotspot. An animal studied showed that Breg subpopulations that secrete IL-10 $\left(\mathrm{CD} 1 \mathrm{~d}^{\text {hi }} \mathrm{CD}^{+}, \mathrm{B} 10\right)$ can effectively inhibit the proliferation of effector $\mathrm{T}$ cells and alleviate the decline in $\beta$-cell function by secret IL-10 (32). Breg cells were increased in the early recovery stage of T1DM (15), and the B10 subgroup was positively correlated with fasting C-peptide level and negatively correlated with $\mathrm{HbA1c}$ of T1DM patients (30). The evidence suggests that B-cell subsets participate in the process of the remission phase. In addition, it was reported that $\mathrm{B} 10$ cells can reduce $\mathrm{T}$ cell-mediated islet transplant rejection by promoting Treg-cell development and inhibiting pro-inflammatory Th1-cell activity (33). Considering this situation, it is a high value to investigate the effects and mechanisms possible of B10-cell subsets in the remission phase.

At present, studies about glucose metabolism and immune cells are mainly concentrated in T cells and macrophages. Studies of the relationship between $\mathrm{B}$ cells and glucose metabolism are rare. An in vitro study showed that B-lymphocyte activating factor, lipopolysaccharide, and B-cell receptor rapidly accelerate glucose uptake and glycolysis, which provide rapid energy for cell proliferation (34). In addition, Kojima et al. (35) reported that hypoxia-inducible factors play a part in upregulating glycolysis during B-cell development. Furthermore, hypoxia-inducible factor- $1 \alpha$ plays a critical role in the expansion of B10 cells and the expression of IL-10 (36). It is suggested that the regulation of B-cell energy metabolism is essential for its development and function. Therefore, the rectification of hyperglycemia may affect the normal function of B-cell subsets and participate in the remission phase immune modulation process. However, there is no relevant research evidence yet.

\section{Other Immune Cells}

Besides T and B cells, other immune cells such as natural killer (NK) cells, monocytes, and neutrophils may also participate in the remission phase of T1DM. Fitas et al. (13) reported that the number of neutrophil and NK cells was significantly reduced at the onset of T1DM and began to recover during the remission period, and low percentage of NK cells and high percentage of neutrophils were positively correlated with the duration of the remission phase. The number of mononuclear cells also decreased after the onset of T1DM and reached the nadir in the first year (15). These changes of the immune cells in peripheral blood suggest an active extravasation to target tissues, probably contributing to the downregulation of the autoimmune response (16).

\section{CYTOKINES}

The roles of pro-inflammatory and anti-inflammatory cytokines in the pathogenesis of T1DM have been known for a long time. Numerous cytokines have been tested as possible biomarkers for the remission phase. Hvidoere's team found that Th1-related chemokines CCL5 decreased in patients with remission and was positively correlated with HbA1c, while CCL3 increased in these patients but negatively correlated with C-peptide
(37). Furthermore, patients during the remission phase were accompanied with decreased IFN- $\gamma$ and TGF- $\beta$ levels, and high levels of IL-10 were in parallel with good glycemic control $(15,38$, 39). However, some inconsistent results had been reported, and pro-inflammatory IL- 6 was shown to be elevated in the remission phase while demonstrated a positive correlation with glycemic control (40). When different cytokines were taken together and defined patients according to multiple cytokines, that is, lowresponder patients who did not detect any anti-inflammatory (IL-4, IL-10, and IL-13) and pro-inflammatory factors (TNF- $\alpha$ ) and high-responder patients with at least one cytokine detected, it was found that low responders had higher C-peptide levels and longer remission duration than high responders (13), suggesting the downregulation of pro-inflammation in the remission phase.

Glucose metabolism is implicated in the change pattern of cytokines. Soluble interleukin-7 (IL-7) receptor $\alpha$ (sCD127) expressed on the surface of $\mathrm{T}$ cells, when combined with IL7, has an antagonistic effect on IL-7 signaling pathway and IL-7-mediated T-cell proliferation (41). It is worth noting that hyperglycemia resulted in a glycosylated form of sCD127 that was ineffective as an IL-7 antagonist and glycosylated sCD127 was found in patients with T1DM (42), which provided new evidence that hyperglycemia regulates the immune network by acting on immune molecules.

\section{IMMUNE MOLECULES}

The role of inhibitory immune-related molecules such as CTLA4 is crucial in the pathogenesis of T1DM. Recently, programmed cell death-1 (PD-1), a transmembrane glycoprotein belonging to CD28/CTLA4 family, which is acting as an important immunosuppressive molecule that is mainly expressed on activated $\mathrm{T}$ or $\mathrm{B}$ cells, is regaining the attention in the field of T1DM as well as in oncology (43). It was shown that the infiltration of autoimmune islet inflammation and islet antigen reactive $\mathrm{T}$ cells was aggravated when $\mathrm{PD}-1$ or $\mathrm{PD}-\mathrm{L} 1$ was blocked (44). PD-1/PD-L1 as a therapeutic target can effectively inhibit autoimmune $\mathrm{T}$-cell response in non-obese diabetic mice and reverse diabetes (23). The widespread application of PD-1/PDL1 antibody therapy is an epoch-making event in the field of oncology, but their side effects as PD-1 inhibitor induced T1DM have prompted us to explore the roles of PD-1/PD-L1 in T1DM and its possible participation in the remission phase. It is noteworthy that the expression of PD-L1 on $\beta$ cells could alleviate autoimmune attacks and resist T-cell-mediated destruction (45, 46), making PD-L1 as a potential biomarker for recognizing $\beta$ cells that resist the immune attack. In this sense, it is of particular interest to explore whether PD-1/PD-L1 expression of immune cells and $\beta$ cells could be a predictor for the occurrence and duration of the remission phase.

\section{IMMUNOGENICITY OF ISLET $\beta$ CELLS}

A study showed that some $\beta$ cells can survive a long time without being immune attacked by reducing the expression of diabetesassociated antigens IGRP, Chga, Gad1, and Ins1/Ins2 (46). This 
TABLE 1 | The immunological changes during the different stage of the remission phase.

\begin{tabular}{|c|c|c|}
\hline Immunological markers & $\begin{array}{l}\text { Changes during the } \\
\text { remission phase }\end{array}$ & References \\
\hline $\begin{array}{l}\text { aTreg, Th17, Breg, } \\
\text { Neutrophil, CCL3, IL-6 }\end{array}$ & $\begin{array}{l}\text { Increased at the beginning } \\
\text { of the remission phase }\end{array}$ & $(13,15,37,40)$ \\
\hline $\begin{array}{l}\text { B cells, mononuclear, NK } \\
\text { cells, CCL5, INF- } \gamma \text {, TGF- } \beta \text {, } \\
\beta \text {-cell immunogenicity, }\end{array}$ & $\begin{array}{l}\text { Decreased at the beginning } \\
\text { of the remission phase }\end{array}$ & $(13,15,37,38,52)$ \\
\hline $\begin{array}{l}\text { aTreg; } \\
\text { CD4 }{ }^{+} \mathrm{CD} 25^{+} \mathrm{CD} 127^{\text {hi }} \text { cells; } \\
\text { CD4+CD45RO }{ }^{+} \text {memory } \\
\text { cells; Neutrophil; IL-10, }\end{array}$ & $\begin{array}{l}\text { Positively correlation with } \\
\text { the length of the remission } \\
\text { phase }\end{array}$ & $(13,14,39)$ \\
\hline NK cells; IL-4; IL-13; TNF- $\alpha$, & $\begin{array}{l}\text { Negatively correlation with } \\
\text { the length of the remission } \\
\text { phase }\end{array}$ & (13) \\
\hline
\end{tabular}

suggests that changes of $\beta$-cell antigenicity play an important role in the immune destruction process. The effect of blood glucose on the immunogenicity of $\beta$ cells has long been documented, and a hyperglycemic environment could stimulate antigen expression (47-49). A new study found that resident macrophages in islets could take up the $\beta$ endocellular insulin-containing granules through its filopodial and present insulin peptides to insulinreactive $\mathrm{T}$ cells and the antigen presentation was increased after high-glucose stimulation in non-obese diabetic mice (50). Skowera et al. (51) showed that the expression of cell-specific proinsulinogen (PPI) signaling epitopes increased in longterm hyperglycemic concentrations, leading to increased PPIspecific CD8+ T-cell killing of cells and accelerated $\beta$-cell death. Accordingly, a significant decrease of autoantigen expression of $\beta$ cells was observed after insulin treatment $(52,53)$. Taken together, it was deemed that correction of the "glucotoxicity" during the remission phase may downregulate immune response by reducing the immunogenicity of $\beta$ cells.

\section{DISCUSSION}

To sum up, patients in remission phase were accompanied by changes in the frequency of various cells and immune molecules,

\section{REFERENCES}

1. Li X, Cheng J, Zhou Z. Revisiting multiple models of progression of $\beta$-cell loss of function in type 1 diabetes: significance for prevention and cure. J Diabetes. (2016) 8:460-9. doi: 10.1111/1753-0407.12376

2. Atkinson MA, Roep BO, Posgai A, Wheeler DCS, Peakman M. The challenge of modulating $\beta$-cell autoimmunity in type 1 diabetes. Lancet Diabetes Endocrinol. (2019) 7:52-64. doi: 10.1016/S2213-8587(18)3 0112-8

3. Zhong T, Tang R, Gong S, Li J, Li X, Zhou Z. The remission phase in type 1 diabetes: changing epidemiology, definitions, and emerging immuno-metabolic mechanisms. Diabetes Metab Res Rev. (2019) 2019:e3207. doi: 10.1002/dmrr.3207

4. Jackson RL, Boyd JD, Smith TE. Stabilzation of the diabetic child. Proc Soc Exp Biol Med Soc Exp Biol Med. (1940) 157:610-4. including increased aTreg, Th17, Breg, and neutrophil cells to varying degrees, decreased B cells, NK cells, IFN- $\gamma$, and lower $\beta$ cell immunogenicity, partly due to hyperglycemia rectification. Meanwhile, duration of the remission phase is also related to a variety of cells and molecules, such as aTreg, CD $25^{+} \mathrm{CD} 127 \mathrm{hi}$ cells, $\mathrm{CD}_{45 \mathrm{RO}^{+}}$memory cells, neutrophil, and IL-10, which are positively correlated, and NK cells and some inflammatory molecules (IL-4, IL-10, IL-13, and TNF- $\gamma$ ), which are negatively correlated (Table 1). The occurrence of remission phase is based on the immune modulation as well as $\beta$-cell rest, and rectification of hyperglycemia is likely to be the main cause of this immune change. Although the exact mechanisms are not clear, it could be deemed that controlling hyperglycemia not only is important to $\beta$-cell rest but also has vital effects on various immune components. The best way to intervene in T1DM may be to combine strict glycemic control with immune intervention to protect $\beta$-cell functions as early as possible and as long as possible.

Although there are several published studies on the remission phase of T1DM $(54,55)$, reviews focusing on mechanisms of immune modulation by hyperglycemia rectification are lacking. Our review has some strength in providing a comprehensive overview and new evidence on the interaction between glucose metabolism and immune regulation from multiple aspects such as immune cells, inflammatory cytokines, biomolecules, and cell antigenicity. The importance of immune-glycometabolism should be emphasized in the pathogenesis of the remission phase.

\section{AUTHOR CONTRIBUTIONS}

RT wrote the manuscript. TZ contributed to the Cytokines and Immune molecules section. CW searched part of the references. $\mathrm{XL}$ provided ideas. ZZ polished the article.

\section{FUNDING}

This work was supported by the National Key R\&D Program of China (Grant Nos. 2016YFC1305000, 2016YFC1305001, and 2017YFC1309604) and the Natural Science Foundation of Hunan Province, China (Grant No. 2019JJ40419). 67:429-44. doi: 10.1001/archpedi.1944.02020060002001

6. Best $\mathrm{CH}$, Campbell J, Haist RE. The effect of anterior pituitary extracts on the insulin content of the pancreas. J Physiol. (1939) 97:2006. doi: 10.1113/jphysiol.1939.sp003799

7. Lukens FDW, Dohan FC. Morphological and functional recovery of the pancreatic islands in diabetic cats treated with insulin. Science. (1940) 92:2223. doi: $10.1126 /$ science. 92.2384 .222

8. Ludvigsson J, Heding LG. Beta-cell function in children with diabetes. Diabetes. (1978) 27(Suppl 1):230-4. doi: 10.2337/diab.27.1.S230

9. Shah SC, Malone JI, Simpson NE. A randomized trial of intensive insulin therapy in newly diagnosed insulin-dependent diabetes mellitus. NEngl J Med. (1989) 320:550-4. doi: 10.1056/NEJM198903023200902

10. Chobot A, Stompor J, Szyda K, Sokolowska M, Deja G, Polanska J, et al. Remission phase in children diagnosed with type 1 diabetes in years 2012 
to 2013 in Silesia, Poland: an observational study. Pediatr Diabetes. (2019) 20:286-92. doi: 10.1111/pedi.12824

11. Fonolleda M, Murillo M, Vazquez F, Bel J, Vives-Pi M. Remission phase in paediatric type 1 diabetes: new understanding and emerging biomarkers. Hormone Res Paediatr. (2017) 88:307-15. doi: 10.1159/000479030

12. Aly H, Gottlieb P. The honeymoon phase: intersection of metabolism and immunology. Curr Opin Endocrinol Diabetes Obes. (2009) 16:28692. doi: 10.1097/MED.0b013e32832e0693

13. Fitas AL, Martins C, Borrego LM, Lopes L, Jorns A, Lenzen S, et al. Immune cell and cytokine patterns in children with type 1 diabetes mellitus undergoing a remission phase: a longitudinal study. Pediatr Diabetes. (2018) 19:96371. doi: $10.1111 /$ pedi.12671

14. Moya R, Robertson HK, Payne D, Narsale A, Koziol J, Davies JD. A pilot study showing associations between frequency of $\mathrm{CD} 4(+)$ memory cell subsets at diagnosis and duration of partial remission in type 1 diabetes. Clin Immunol. (2016) 166-167:72-80. doi: 10.1016/j.clim.2016.04.012

15. Villalba A, Fonolleda M, Murillo M, Rodriguez-Fernandez S, Ampudia RM, Perna-Barrull D, et al. Partial remission and early stages of pediatric type 1 diabetes display immunoregulatory changes. A pilot study. Transl Res. (2019) 210:8-25. doi: 10.1016/j.trsl.2019.03.002

16. Oras A, Peet A, Giese T, Tillmann V, Uibo R. A study of 51 subtypes of peripheral blood immune cells in newly diagnosed young type 1 diabetes patients. Clin Exp Immunol. (2019) 198:57-70. doi: 10.1111/cei.13332

17. Mbongue JC, Rawson J, Garcia PA, Gonzalez N, Cobb J, Kandeel F, et al. Reversal of new onset type 1 diabetes by oral salmonella-based combination therapy and mediated by regulatory T-cells in NOD Mice. Front Immunol. (2019) 10:320. doi: 10.3389/fimmu.2019.00320

18. Li Z, Shi X, Liu J, Shao F, Huang G, Zhou Z, et al. Artesunate prevents type 1 diabetes in NOD mice mainly by inducing protective IL-4-producing $\mathrm{T}$ cells and regulatory T cells. FASEB J. (2019) 33:8241-8. doi: 10.1096/fj.201900146R

19. Viisanen T, Gazali AM, Ihantola EL, Ekman I, Nanto-Salonen K, Veijola R, et al. FOXP3 + regulatory $\mathrm{T}$ cell compartment is altered in children with newly diagnosed type 1 diabetes but not in autoantibody-positive at-risk children. Front Immunol. (2019) 10:19. doi: 10.3389/fimmu.2019.00019

20. Patterson SJ, Pesenacker AM, Wang AY, Gillies J, Mojibian M, Morishita $\mathrm{K}$, et al. $\mathrm{T}$ regulatory cell chemokine production mediates pathogenic $\mathrm{T}$ cell attraction and suppression. J Clin Invest. (2016) 126:103951. doi: 10.1172/JCI83987

21. Attias M, Al-Aubodah T, Piccirillo CA. Mechanisms of human FoxP3(+) treg cell development and function in health and disease. Clin Exp Immunol. (2019) 197:36-51. doi: 10.1111/cei.13290

22. Jaberi-Douraki M, Pietropaolo M, Khadra A. Continuum model of Tcell avidity: understanding autoreactive and regulatory T-cell responses in type 1 diabetes. J Theor Biol. (2015) 383:93-105. doi: 10.1016/j.jtbi.201 5.07 .032

23. Maciver NJ, Jacobs SR, Wieman HL, Wofford JA, Coloff JL, Rathmell JC. Glucose metabolism in lymphocytes is a regulated process with significant effects on immune cell function and survival. J Leukocyte Biol. (2008) 84:94957. doi: $10.1189 / \mathrm{jlb} .0108024$

24. Angelin A, Gil-de-Gomez L, Dahiya S, Jiao J, Guo L, Levine $\mathrm{MH}$, et al. Foxp3 reprograms $\mathrm{T}$ cell metabolism to function in low-glucose, high-lactate environments. Cell Metab. (2017) 25:1282-93.e7. doi: 10.1016/j.cmet.2016.12.018

25. Gabriel SS, Kallies A. Sugars and fat - a healthy way to generate functional regulatory $\mathrm{T}$ cells. Eur J Immunol. (2016) 46:2705-9. doi: 10.1002/eji.201646663

26. Cluxton D, Petrasca A, Moran B, Fletcher JM. Differential regulation of human treg and Th17 cells by fatty acid synthesis and glycolysis. Front Immunol. (2019) 10:115. doi: 10.3389/fimmu.201 9.00115

27. Yao Y, Vent-Schmidt J, McGeough MD, Wong M, Hoffman HM, Steiner TS, et al. Tr1 cells, but not Foxp3+ regulatory T cells, suppress NLRP3 inflammasome activation via an IL-10-dependent mechanism. J Immunol. (2015) 195:488-97. doi: 10.4049/jimmunol.1403225

28. Guarda G, Dostert C, Staehli F, Cabalzar K, Castillo R, Tardivel A, et al. $\mathrm{T}$ cells dampen innate immune responses through inhibition of NLRP1 and NLRP3 inflammasomes. Nature. (2009) 460:269-73. doi: 10.1038/nature 08100
29. Habib T, Long SA, Samuels PL, Brahmandam A, Tatum M, Funk A, et al. Dynamic immune phenotypes of B and T helper cells mark distinct stages of T1D progression. Diabetes. (2019) 68:1240-50. doi: 10.2337/db1 8-1081

30. Deng C, Xiang Y, Tan T, Ren Z, Cao C, Huang G, et al. Altered peripheral B-lymphocyte subsets in type 1 diabetes and latent autoimmune diabetes in adults. Diabetes Care. (2016) 39:434-40. doi: 10.2337/dc15-1765

31. Kurozumi A, Okada Y, Arao T, Miyazaki Y, Yoshikawa M, Torimoto K, et al. Pancreas-protective effect of rituximab for acute-onset type 1 diabetes in the honeymoon period: a case report. Endocrinol Diabetes Metab Case Rep. (2016) 2016:160020. doi: 10.1530/EDM-16-0020

32. Candando KM, Lykken JM, Tedder TF. B10 cell regulation of health and disease. Immunol Rev. (2014) 259:259-72. doi: 10.1111/imr.12176

33. Qin $\mathrm{Y}$, Zhang $\mathrm{M}$, Jiang RM, Wu Q, Xu XY, Chen H, et al. B10 cells play a role in the immune modulation of pro- and anti-inflammatory immune responses in mouse islet allograft rejection. Cell Immunol. (2016) 310:18492. doi: 10.1016/j.cellimm.2016.09.010

34. Jellusova J, Rickert RC. The PI3K pathway in B cell metabolism. Crit Rev Biochem Mol Biol. (2016) 51:359-78. doi: 10.1080/10409238.2016.1215288

35. Kojima H, Kobayashi A, Sakurai D, Kanno Y, Hase H, Takahashi R, et al. Differentiation stage-specific requirement in hypoxia-inducible factor- $1 \alpha$ regulated glycolytic pathway during murine $\mathrm{B}$ cell development in bone marrow. J Immunol. (2010) 184:154-63. doi: 10.4049/jimmunol.0800167

36. Meng X, Grotsch B, Luo Y, Knaup KX, Wiesener MS, Chen XX, et al. Hypoxia-inducible factor- $1 \alpha$ is a critical transcription factor for IL-10-producing B cells in autoimmune disease. Nat Commun. (2018) 9:251. doi: $10.1038 / \mathrm{s} 41467-017-02683-\mathrm{x}$

37. Pfleger C, Kaas A, Hansen L, Alizadeh B, Hougaard P, Holl R, et al. Relation of circulating concentrations of chemokine receptor CCR5 ligands to Cpeptide, proinsulin and $\mathrm{HbAlc}$ and disease progression in type 1 diabetes. Clin Immunol. (2008) 128:57-65. doi: 10.1016/j.clim.2008.03.458

38. Alizadeh BZ, Hanifi-Moghaddam P, Eerligh P, van der Slik AR, Kolb H, Kharagjitsingh AV, et al. Association of interferon-gamma and interleukin 10 genotypes and serum levels with partial clinical remission in type 1 diabetes. Clin Exp Immunol. (2006) 145:480-4. doi: 10.1016/j.clim.200 6.04 .465

39. Karges B, Durinovic-Bello I, Heinze E, Debatin KM, Boehm B, Karges W. Immunological mechanisms associated with long-term remission of human type 1 diabetes. Diabetes Metab Res Rev. (2006) 22:1849. doi: $10.1002 / \mathrm{dmrr} .600$

40. Pfleger C, Mortensen HB, Hansen L, Herder C, Roep BO, Hoey $\mathrm{H}$, et al. Association of IL-1ra and adiponectin with C-peptide and remission in patients with type 1 diabetes. Diabetes. (2008) 57:92937. doi: $10.2337 / \mathrm{db} 07-1697$

41. Monti P, Scirpoli M, Maffi P, Ghidoli N, De Taddeo F, Bertuzzi F, et al. Islet transplantation in patients with autoimmune diabetes induces homeostatic cytokines that expand autoreactive memory T cells. J Clin Invest. (2008) 118:1806-14. doi: 10.1172/JCI35197

42. Monti P, Brigatti C, Krasmann M, Ziegler AG, Bonifacio E. Concentration and activity of the soluble form of the interleukin-7 receptor $\alpha$ in type 1 diabetes identifies an interplay between hyperglycemia and immune function. Diabetes. (2013) 62:2500-8. doi: 10.2337/db12-1726

43. Pauken KE, Jenkins MK, Azuma M, Fife BT. PD-1, but not PD-L1, expressed by islet-reactive $\mathrm{CD} 4+\mathrm{T}$ cells suppresses infiltration of the pancreas during type 1 diabetes. Diabetes. (2013) 62:2859-69. doi: 10.2337/db1 2-1475

44. Ansari MJ, Salama AD, Chitnis T, Smith RN, Yagita H, Akiba $H$, et al. The programmed death-1 (PD-1) pathway regulates autoimmune diabetes in nonobese diabetic (NOD) mice. J Exp Med. (2003) 198:639. doi: $10.1084 /$ jem. 20022125

45. Colli ML, Hill JLE, Marroqui L, Chaffey J, Dos Santos RS, Leete P, et al. PDL1 is expressed in the islets of people with type 1 diabetes and is upregulated by interferons- $\alpha$ and- $\gamma$ via IRF1 induction. EBioMedicine. (2018) 36:367-75. doi: 10.1016/j.ebiom.2018.09.040

46. Rui J, Deng S, Arazi A, Perdigoto AL, Liu Z, Herold KC. Beta cells that resist immunological attack develop during progression of autoimmune diabetes in NOD mice. Cell Metab. (2017) 25:727-38. doi: 10.1016/j.cmet.201 7.01 .005 
47. Buschard K, Brogren $\mathrm{CH}$, Ropke C, Rygaard J. Antigen expression of the pancreatic beta-cells is dependent on their functional state, as shown by a specific, BB rat monoclonal autoantibody IC2. APMIS: Acta Pathologica, Microbiologica, et Immunologica Scandinavica. (1988) 96:342-6. doi: 10.1111/j.1699-0463.1988. tb05313.x

48. Bjork E, Kampe O, Karlsson FA, Pipeleers DG, Andersson A, Hellerstrom C, et al. Glucose regulation of the autoantigen GAD65 in human pancreatic islets. J Clin Endocrinol Metab. (1992) 75:1574-6. doi: 10.1210/jcem.75.6. 1464667

49. Hagopian WA, Karlsen AE, Petersen JS, Teague J, Gervassi A, Jiang J, et al. Regulation of glutamic acid decarboxylase diabetes autoantigen expression in highly purified isolated islets from Macaca nemestrina. Endocrinol. (1993) 132:2674-81. doi: 10.1210/endo.132.6. 8504767

50. Zinselmeyer BH, Vomund AN, Saunders BT, Johnson MW, Carrero JA, Unanue ER. The resident macrophages in murine pancreatic islets are constantly probing their local environment, capturing beta cell granules and blood particles. Diabetologia. (2018) 61:1374-83. doi: 10.1007/s00125-018-4592-4

51. Skowera A, Ellis RJ, Varela-Calvino R, Arif S, Huang GC, VanKrinks C, et al. CTLs are targeted to kill beta cells in patients with type 1 diabetes through recognition of a glucose-regulated preproinsulin epitope. J Clin Invest. (2008) 118:3390-402. doi: 10.1172/jci 35449
52. Brown RJ, Rother KI. Effects of beta-cell rest on beta-cell function: a review of clinical and preclinical data. Pediatr Diabetes. (2008) 9:14-22. doi: 10.1111/j.1399-5448.2007.00272.x

53. Marre ML, James EA, Piganelli JD. Beta cell ER stress and the implications for immunogenicity in type 1 diabetes. Front Cell Developmental Biology. (2015) 3:67. doi: 10.3389/fcell.2015.00067

54. Chetan MR, Charlton MH, Thompson C, Dias RP, Andrews RC, Narendran P. The type 1 diabetes 'honeymoon' period is five times longer in men who exercise: a case-control study. Diabetic Med. (2019) 36:127-8. doi: 10.1111/dme.13802

55. Moosavi M, Seguin J, Polychronakos C. Effect of autoimmunity risk loci on the honeymoon phase in type 1 diabetes. Pediatric Diabetes. (2017) 18:459-62. doi: $10.1111 /$ pedi. 12421

Conflict of Interest: The authors declare that the research was conducted in the absence of any commercial or financial relationships that could be construed as a potential conflict of interest.

Copyright $\odot 2019$ Tang, Zhong, Wu, Zhou and Li. This is an open-access article distributed under the terms of the Creative Commons Attribution License (CC BY).

The use, distribution or reproduction in other forums is permitted, provided the original author(s) and the copyright owner(s) are credited and that the original publication in this journal is cited, in accordance with accepted academic practice. No use, distribution or reproduction is permitted which does not comply with these terms. 